\title{
Die Naturheilpraktikerin Pauline Felder - eine biographische Annäherung an schweizerische Volksmedizin im 20. Jahrhundert
}

\author{
Sandra Clair ${ }^{\mathrm{a}}$ Reinhard Saller ${ }^{\mathrm{b}}$ \\ Institut für Naturheilkunde, Universitätsspital Zürich, Zürich, Schweiz
}

\section{Schlüsselwörter \\ Pauline Felder - Ordensschwester - Hebamme - Naturheilpraktikerin . Naturheilkunde - Schweizerische Volksmedizin · Phytotherapie . \\ Traditionelle Europäische Medizin · Medizingeschichte}

\section{Zusammenfassung}

Die franziskanische Ordensschwester Pauline Felder (1925-2007) ist eine der bekanntesten schweizerischen Naturheilkundlerinnen der jüngsten Vergangenheit. An ihrem Beispiel wird exemplarisch aufgezeigt, dass die schweizerische Volksmedizin eine lebende Tradition ist, die komplementär zu naturwissenschaftlichen Methoden der Biomedizin einen wichtigen Beitrag zur ganzheitlichen Gesundheitsversorgung der lokalen Bevölkerung beiträgt. Dieser Artikel beleuchtet Schwester Paulines familiäre und religiöse Sozialisation, ihre berufliche Ausbildung zur Hebamme, ihr gewähltes Leben als Nonne sowie ihren Werdegang zur Naturheilpraktikerin. Ihre therapeutischen Anwendungen basierten vornehmlich auf mündlich tradiertem phytotherapeutischen Wissen, welches ihr als integraler Teil der Gesundheitsversorgung während ihrer Kindheit und Jugend vermittelt worden war, weiter auf ihrer medizinischen Fachausbildung in den 1940er-Jahren, die nach wie vor naturheilkundliche Pflegeanwendungen lehrte, sowie literarischen und praktischen Fortbildungen. Ab den 1980er-Jahren erweiterte sie ihre phyto- und hydrotherapeutische Arbeitsweise mit zum Teil unorthodoxen Methoden. In zahlreichen Vorträgen, Kursen, Interviews, schriftlichen Erzeugnissen sowie als Mentorin von Praktikantinnen und Praktikanten verbreitete sie ihr Wissen und trug damit zur Erhaltung der schweizerischen Volksmedizin bei. Ihre jährlich selbst zubereiteten Heilmittel, die sie mittels traditioneller Sammel- und Verarbeitungsregeln herstellte, hatten bei ihrer Kundschaft die Reputation, von besonders guter Qualität zu sein. Der langjährige Erfolg ihrer Gesundheitsberatungsstelle zeigt beispielhaft, dass parallel zur Schulmedizin Heilmittel der Traditionellen Europäischen Medizin bei Patientinnen und Patienten erwünscht sind. Er illustriert auch, dass psychosoziale Aspekte für eine erfolgreiche Behandlung von elementarer Bedeutung sind.

(c) 2015 S. Karger GmbH, Freiburg

\section{Keywords}

Pauline Felder · Nun · Midwife · Naturopath · Naturopathy · Swiss folk medicine - Phytotherapy - Traditional European Medicine . History of medicine

\section{Summary}

The Naturopath Pauline Felder - a Biographic Approach to Swiss Folk Medicine in the 20th Century

The Franciscan nun Sister Pauline Felder (1925-2007) is one of the most well-known Swiss natural medicine practitioners of the recent past. Her example demonstrates that Swiss folk medicine is a living tradition that complements the natural science methods of biomedicine and offers an important contribution to the holistic healthcare of the local population. The article illustrates her familial and religious socialisation, her professional education as a midwife, her chosen life as a nun as well as her career as a natural medicine practitioner. Her therapeutic applications were mainly based on orally transmitted knowledge of phytotherapy, to which she was exposed during her childhood and adolescence as an integral part of contemporary healthcare. They were further grounded in her medical training in the 1940s when naturopathic care was still taught, and fostered during her further literary and practical education. From 1980 onwards she expanded her phyto- and hydrotherapeutic practices to include some unorthodox methods. In numerous lectures, seminars, interviews, written documents, and as a mentor of interns she disseminated her knowledge and contributed to the preservation of Swiss folk medicine. Her remedies, which she prepared annually employing traditional rules of collection and compounding, had the reputation among her clientele as being of particularly good quality. The enduring success of her advice centre for natural healthcare confirms that parallel to biomedicine, patients seek remedies from Traditional European Medicine. It also illustrates that psychosocial aspects of the therapeutic encounter are additionally of elemental significance for healing.

\section{KARGER \\ Fax +497614520714}

(C) 2015 S. Karger GmbH, Freiburg

$1015-0684 / 15 / 0276-0354 \$ 39.50 / 0$ 


\section{Einleitung}

Die moderne Phytotherapie ist in zunehmendem Mass geprägt durch die Methodik von Naturwissenschaft und Biomedizin und ist zu einer systematischen Dokumentation von objektiv quantifizierbaren Daten aufgefordert. In einer Zeit, da evidenzbasierte Medizin zum neuen Lehrsatz der Heilkunst erhoben worden ist, erscheint es lohnenswert, auf die Wurzeln der empirisch geprägten Natur- und Pflanzenheilkunde zu blicken, um zu sehen, auf welcher Basis traditionell praktiziertes Heilen von Krankheiten vollzogen wurde und zu welcher Resonanz es bei Patientinnen und Patienten führen kann, wenn es kenntnisreich, reflektiert und verantwortungsvoll eingesetzt wird.

Im Folgenden soll versucht werden, das Wirken der Ordensschwester Pauline Felder (1925-2007) - einer der bekanntesten schweizerischen Naturheilkundlerinnen der jüngsten Vergangenheit - nachzuzeichnen. Der vorliegende Artikel illustriert den Lebenslauf und das Werk dieser originellen Vertreterin der Traditionellen Europäischen Naturheilkunde basierend auf bibliographischen Angaben in ihren Büchern, Interviews sowie persönlichen Beobachtungen während eines längeren Praktikums von Anfang 1991 bis Ende 1993, das die Erstautorin als Teil ihres ethnomedizinischen Studiums an der Universität Bern bei Pauline Felder absolvierte.

\section{Familiäre Sozialisation}

Pauline Felder wurde am 16. Januar 1925 in Schüpfheim in eine religiöse Handwerksfamilie geboren und wuchs mit ihrer älteren Schwester Maria Magdalena und ihrem jüngeren Bruder Johann Josef im streng katholischen Entlebuch, Kanton Luzern, auf. Die bescheidenen Verhältnisse und harten Kriegsjahre verlangten von ihr schon früh ein tatkräftiges und selbstloses Anpacken im familiären Haushalt und in der Nachbarschaft - eine Prägung, die Pauline lebenslang zum praktischen Handeln veranlassen sollte.

Ihre Mutter Agatha Felder, geborene Dahinden, war vor ihrer Heirat mit dem Schuhmacher Gottfried Felder aus Schüpfheim Vorarbeiterin in der Uhrenfabrik Kummer im solothurnischen Bettlach. Unter dem eher unduldsamen und gefühlsarmen Vater litt die junge Pauline, sodass sie viel Zeit mit ihrer musikalischen Mutter und deren naturliebenden Verwandtschaft verbrachte. Das Sammeln von Kräutern, Beeren und Früchten in der freien Natur gehörte ebenso zu ihrer Jugend wie das herzhafte Singen von Volksliedern zur Erbauung des Gemüts.

Die tiefe Religiosität der Eltern beeinflusste ihr Verständnis der Natur. Aus der Schöpfungsgeschichte entnahm sie, dass Pflanzen ein Geschenk seien, welches Gott als Lebensvoraussetzung für Menschen und Tiere geschaffen habe. Diese Wunderwerke sollten nicht nur als Nahrungsmittel, Baumaterial und Kleiderstoffe, sondern auch als Heilmittel zur Erhaltung der Gesundheit verwendet werden.

Der volksheilkundliche Gebrauch von Kräutern war in Paulines Kindheit und Jugend ein integraler Teil der lokalen Gesundheitsversorgung. Die am eigenen Leib erlebten oder sonst beobachteten Anwendungen traditioneller Heilpflanzen legten den Grundstein für das unerschütterliche Vertrauen, das Pauline in die Naturmedizin legte.

\section{Berufliche und religiöse Grundlagen}

Pauline fiel in der Primarschule durch Wissensdurst, Intelligenz, unabhängiges Denken und ein gutes Gedächtnis auf. Statt des ersehnten Übertritts in die Sekundarschule zwang die familiäre wirtschaftliche Not sie jedoch, die Schule in der siebten Klasse im Alter von 14 Jahren zu verlassen, um bei einer kinderreichen Nachbarin den Haushalt zu führen. Ein Stipendium ermöglichte ihr 1942 im Alter von 17 Jahren den Besuch einer Haushaltsschule im luzernischen Sursee. Diese formelle Ausbildung verhalf ihr zu ihrer ersten regulären Stelle im Bürgerheim in Menznau, einer kleinen Willisauer Gemeinde mit knapp 2500 Einwohnern. Hier begeisterte die fröhliche Pauline vom ersten Tag an die 70-köpfige Gemeinschaft von Betagten und Behinderten mit ihrem herzhaften Singen. Nach Menznau folgten weitere Stellen in verschiedenen ländlichen Haushalten ihrer Heimatgemeinde Schüpfheim, bis sie schliesslich als 23-Jährige im Frauenspital Basel eine Ausbildung zur Hebamme antrat.

Der Schritt zu einer von ihren eigenen Ersparnissen bezahlten medizinischen Fachausbildung zur Hebamme dokumentiert Paulines starken Wunsch nach Eigenständigkeit. Gleichzeitig verspürte sie in ihren frühen Zwanzigern immer stärker den inneren Ruf, ihr Leben «in den Dienst des Schöpfers und der Schöpfung» zu stellen. Diese Zeit war gekennzeichnet von einer Auseinandersetzung zwischen den gegensätzlichen Wünschen, eine eigene Familie zu gründen oder ins Kloster einzutreten. Sie lehnte mehr als einen Heiratsantrag ab und verpflichtete sich schliesslich probeweise kurz vor dem Eintritt in die Hebammenschule zu einem Noviziat als Tertianerin des heiligen Franziskus, einer einjährigen Vorschulung für Klosterleute.

Die Jahre ihrer Fachausbildung in Basel waren eine prägende und bereichernde Zeit, verbanden sich in dieser Periode doch gleich mehrere von Paulines Interessen. In den 1940er-Jahren gehörten naturheilkundliche Mittel nach wie vor zum breiten Repertoire einer Schweizer Hebamme und waren somit ein normaler Teil dieses medizinischen Heilberufs [1]. Neben der gynäkologisch orien- 
tierten Hebammenausbildung besuchte sie freiwillig die im Kinderspital gehaltenen Vorlesungen über Ernährung und Kinderkrankheiten. Wöchnerinnen-, Säuglings- und Kleinkinderpflege begeisterten sie in dieser Zeit des intensiven Lernens ebenso wie das Arbeiten im Labor.

Des Weiteren waren ihre Kommilitoninnen aufgeweckte junge Frauen, mit denen sie debattieren wie auch ihren jugendlichen Übermut zeitweilig auslassen konnte. Als Katholikin war sie in der Minderheit und somit gefordert, sich einerseits zu behaupten, sich aber andererseits auch in überkonfessioneller Toleranz zu üben. Diese Erfahrung stellte die Weichen zu ihrer lebenslangen undogmatischen Lebensphilosophie.

Während ihrer Berufsausbildung setzte sie sich auch intensiv mit spirituellen Fragen auseinander [2, S. 29]. Das Johannes-Evangelium wie auch ein ausgeliehenes Buch über das Leben der heiligen Theresia von Lisieux (18731897) waren richtungsweisend. In Paulines Worten: «Der hl. Theresias einfacher Weg zu Gott war mir Wegweiser. Anstatt die steilen Stufen der Vollkommenheit zu erklimmen, nimmt man einfach den Lift: Und das ist die Liebe, Punkt!! Das können alle tun. Ich versuche es auf jeden Fall» [2, S. 33]. Beeinflusst von ihrer religiösen Erziehung im Elternhaus und ihrem Herzenswunsch, ihren Mitmenschen hilfreich zur Seite zu stehen, nahm sie schliesslich nach einem einjährigen Noviziat ihre Lebensweihe als franziskanische Nonne entgegen. Auch wenn dieser Übertritt in einen streng geführten religiösen Orden für die junge und willensstarke Frau nicht selten eine Herausforderung war, übergab sie zum Ende ihrer Hebammenausbildung ihr Leben «der göttlichen Vorsehung» 2 , S. 66] und legte als 26-Jährige das Gelöbnis zu Ehelosigkeit, Armut und Gehorsam ab. Bereits ein paar Monate zuvor war sie im Jahr 1951 in die franziskanische Schwesterngemeinschaft Seraphisches Liebeswerk Solothurn (SLS) übergesiedelt und stellte ihr Leben «mit Hingabe, Freude und steter Verfügbarkeit» [3] in den Dienst für Kinder und Familien in den vom SLS getragenen Mütter-, Kinder- und Pflegeheimen.

Als Ordensfrau verstand Schwester Pauline ihren Dienst am Mitmenschen als Dienst an Gott, der ihre persönliche und spirituelle Kraftquelle war. Gleichzeitig sah sie die Natur als seine für die Menschen geschaffene materielle Kraftquelle zur Stärkung von «Seele, Geist und Körper» [2, S. 63].

\section{Die Wald- und Wiesenhebamme}

Die prägendste Zeit von Schwester Paulines beruflicher Laufbahn war jene als Hebamme im abgelegenen jurassischen Dorf Belfond von 1952 bis 1968. Das Mütternheim war 1952 vom SLS eigens im Hinblick auf ihre Tätigkeit eingerichtet worden und diente als Geburtshaus für junge ledige Frauen, die hier unter Ausschluss einer kritisierenden und verständnisarmen Öffentlichkeit ihre Kinder zur Welt bringen konnten. Als einzige Hebamme und mit einem für die Geburten zugeteilten, aber überlasteten Dorfarzt, der sich vor Geburten scheute, meisterte sie, nicht selten auf sich allein gestellt, 50-70 Geburten pro Jahr. In diesem Zeitraum leistete Schwester Pauline Geburtshilfe bei etwa 1000 Kindern und war des Weiteren für die Nachbetreuung von Mutter und Kind verantwortlich [4].

Da kein externes Labor zur Verfügung stand, gehörten essenzielle Untersuchungen wie Urinproben und Rhesusfaktor-Analyse sowie der Unterhalt der Instrumente in ihren Arbeitsbereich. Auch um die medikamentöse Versorgung ihrer Schützlinge musste sie sich im knapp begüterten Mütternheim oft selbst kümmern. Nicht selten mangelte es an pharmazeutischen Arzneimitteln, sodass sie ihre in der Kindheit und an der Hebammenschule erlernten naturheilkundlichen Kenntnisse zum Einsatz brachte. Ihre zunehmende praktische Erfahrung verfestigte ihr Wissen zur erfolgreichen therapeutischen Anwendung von Heilpflanzen. Schwester Pauline reflektierte viele Jahre später in Solothurn in ihrer Gesundheitsberatungsstelle über diese Zeit [1, S. 9-10]:

«Meine Liebe zur Natur, mein Interesse, selber auszuprobieren, was andere erforscht haben, mein starker Wunsch zu helfen, ohne nach Bezahlung zu fragen, liessen mich immer wieder finden, was ich brauchte, um akute Not zu lindern. Der Grundstein zu meiner heutigen Beratertätigkeit dürfte schon früh gelegt worden sein, nämlich in den fünfzehn Jahren, die ich im Jura verbrachte. Dort arbeitete ich als Hebamme in einem Hause für alleinstehende Mütter und deren Kinder. Abseits von allem, was im städtischen Alltag ablenkt, lebte ich dort in einer herrlich entspannten Atmosphäre, in einer gesunden und intakten Umwelt, naturverbunden, der Schöpfung nahe. Damals galt es noch, den hippokratischen Eid abzulegen, bevor die Erlaubnis zur Berufsausübung erteilt wurde. Dies bedeutete, dass eine Hebamme jederzeit präsent sein muss, Tag und Nacht abrufbereit, für Arm und Reich. So tat ich dort meine Pflicht mit grosser innerer Freude und mit viel Elan. Dabei lernte ich, dass der Schöpfer unendlich viele Kräfte in die Natur gelegt hat, die wir nutzen dürfen, können, sollen.»

Ihre beträchtliche Arbeitslast erledigte sie mithilfe einer praktischen Lebensphilosophie: «Jede Arbeit wird klein, wenn man sie in Stücke zerlegt und immer nur das Nächstliegende vornimmt» [2, S. 39]. Aktive Fürsorge stand ihr näher als passive Fürbitte. Wann immer sie die Zeit dafür fand, sammelte Schwester Pauline in freier Natur heilkräftige Kräuter, die in der Nähe an den über 600 Meter hoch gelegenen Geländeterrassen am östlichen Talhang des Doubstals reichlich vorhanden waren. Die von ihr als «Kräutergarten Gottes» betrachtete Umgebung wurde zu Schwester Paulines grüner Apotheke. Gezielt setzte die «Wald- und Wiesenhebamme» Heilpflanzen ein, so z.B. zur Linderung von Schmerzen das selbstgemachte Johannisöl, zur reichlichen und nahrhaften Milchbildung eine Mischung aus Fenchelfrüchten, Dill- 
früchten, Brennnesselkraut und Himbeerblättern oder zur Hautpflege der Säuglinge eine entzündungshemmende und wundheilende Salbe aus Ringelblumenblüten, Kamillenblüten und Johanniskraut. Ein geburtsstärkender Sirup aus Frauenmantelblättern, Brennnesselkraut, Minzblättern, Schlüsselblumen und Fenchelfrüchten oder ein Heiltee mit Schafgarbenblüten und Johanniskraut für die Wöchnerinnen zur Reinigung und Rückbildung der Gebärmutter gehörten fortan zum Alltag des Mütterheims. So blieben die Gebärenden trotz des Mangels an pharmazeutischen Medikamenten nicht nur vom Wochenbettfieber, sondern auch von anderen schwangerschaftsbedingten Beschwerden verschont [2, S. 41-42]. Gerne gab sie ihre Erfahrungen an junge Praktikantinnen während deren Aufenthalt im Mütternheim weiter und trug so zur Erhaltung des traditionellen naturheilkundlichen Wissens in der Geburtsheilkunde bei.

Auch das seelische Wohl der alleinstehenden Mütter lag Schwester Pauline am Herzen. So war sie nicht nur ihre Hebamme, sondern auch eine Freundin und heiterte die jungen Frauen gerne mit Jodeln, Singen, Gitarrenspiel oder Theater auf. Mit einigen blieb sie in lebenslangem Kontakt, wurde Patin und betreute später in der Gesundheitsberatungsstelle Solothurn deren nun ihrerseits schwangere Töchter mit naturheilkundlichen Mitteln.

Schwester Pauline liebte ihre Tätigkeit in Belfond (Abb. 1, 2). Jedoch machten sich 15 Jahre fast ununterbrochenen Dienstes mit langen Präsenzzeiten und unter oftmals schwierigen Bedingungen mit einer zunehmenden Erschöpfung bemerkbar, sodass sie schliesslich bei den Ordensoberen um eine Ablösung bat, die ihr 1968 gewährt wurde.

\section{Die singende Kräuterfrau}

Nach ihrer Zeit in Belfond arbeitete Schwester Pauline weiterhin vornehmlich in der Mütter- und Kinderbetreuung, jedoch nicht mehr primär als Hebamme, sondern als Krankenpflegerin von erholungsbedürftigen Kindern. Ihre Einsätze in der vom SLS getragenen Villa Teresina in Bombinasco, Tessin, welche 1930 von der Franziskanischen Schwesterngemeinschaft als Kinderpräventorium eröffnet worden war und ab 1979 auch als kostengünstiges Ferien- und Erholungsheim für Familien und Alleinstehende zur Verfügung stand, gefielen ihr besonders gut. Im sonnengetränkten Malcantone sammelte sie, immer von einer Kinderschar begleitet, verschiedenste Heilkräuter zur phytotherapeutischen Behandlung der anvertrauten Mädchen und Jungen. Ihre naturheilkundlichen Erfolge beim Heilen von so diversen Beschwerden wie Bettnässen, Fusspilz oder Asthma sprachen sich herum, sodass Schwester Paulines Ruf als heilkräftige Nonne immer stärker wurde. Sie hatte einen besonderen Draht zu

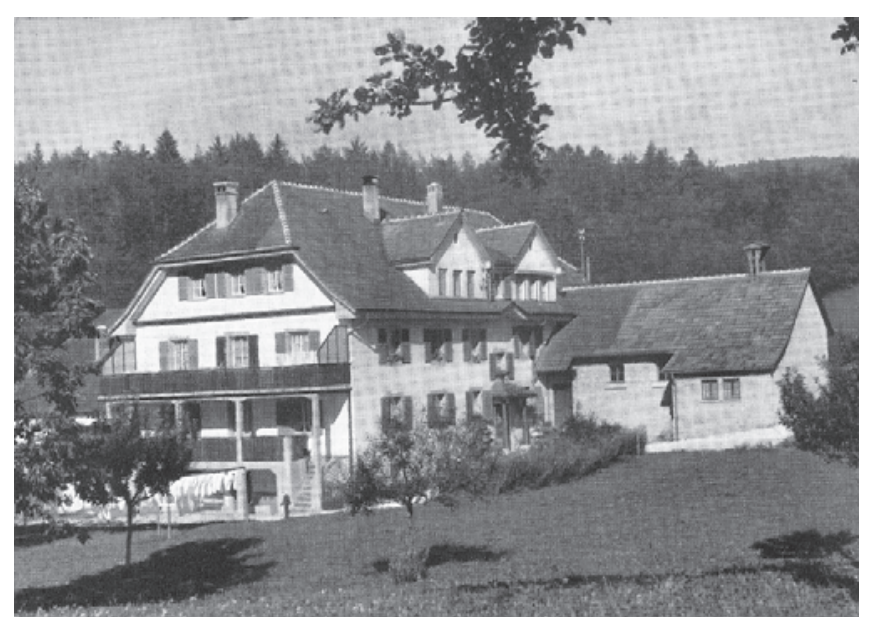

Abb. 1. Das Waisenhaus Foyer Saint-Joseph in Belfond, das vom Seraphischen Liebeswerk Solothurn 1952 im Hinblick auf Schwester Paulines Ankunft als Hebamme als Geburtshaus für junge ledige Mütter («filles-mères») einrichtet wurde (Copyright: Seraphisches Liebeswerk Solothurn).

Abb. 2. Schwester Pauline bei einer Taufe in der Hauskapelle Belfond. Von 1952 bis 1968 betreute sie, oftmals alleine, über 1000 Geburten (Copyright: Seraphisches Liebeswerk Solothurn).

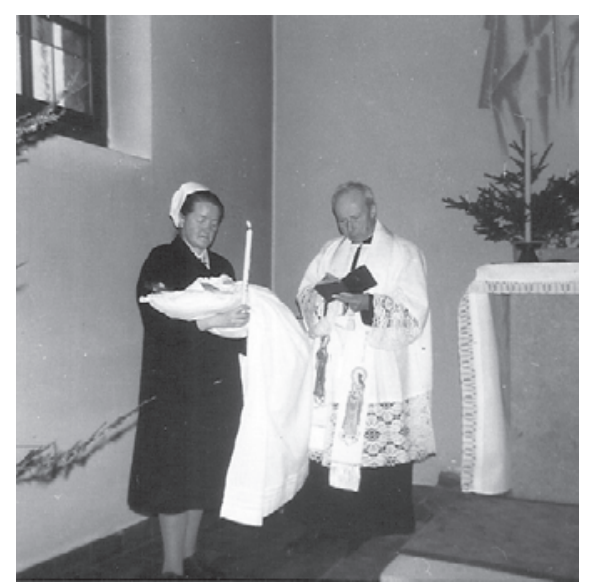

den sensiblen und feinfühligen Kindern, denen sie neben der phytotherapeutischen Betreuung durch Zuspruch das Selbstvertrauen stärkte.

In der stattlichen Bibliothek der 1929 gebauten Villa hatte Schwester Pauline Zugang zu sowohl zeitgenössischen als auch jahrhundertealten, seltenen Kräuterbüchern ärztlicher und volksmedizinischer Autoren. Diese inspirierten sie hinsichtlich der praktischen Anwendung von Heilpflanzen. Während eines Zeitraums von 8 Jahren veröffentlichte sie - ermuntert von ihrem geistlichen Beistand - in der katholischen Zeitschrift St. Josephsblatt die monatliche Kolumne «Gesund bleiben - gesund werden», in der sie ihre Erfahrungen dokumentierte. Dies war der Beginn ihrer Schreibtätigkeit. In diese Zeit fiel auch die Tonbandaufnahme ihrer musikalischen Sammlung von 40 Volksliedern durch das private Tonstudio OberdorfStans, womit sich Schwester Pauline als «singende Kräuterfrau» einen Namen machte [2, S. 60-62].

In der intakten Natur von Bombinasco verbrachte Schwester Pauline gerne ihre Sommerferien, bis das Heim 
1993 zu ihrem grossen Bedauern aus personellen Gründen schliessen musste. In diesem tessinischen Biotop sammelte sie nämlich besonders gerne heilkräftige Kräuter für ihre in der Zwischenzeit eingerichtete Gesundheitsberatungsstelle in Solothurn. Zusammen mit ihren regulären Assistentinnen und Assistenten sowie freiwilligen Helferinnen und Helfern aus der ganzen Schweiz verarbeitete sie diese frisch und unter fröhlichem Singen zu verschiedenen Naturheilmitteln wie Salben, Heilpflanzenölen und Tinkturen oder trocknete sie an der Luft in einem der warmen, schattigen Gebäude zur späteren Herstellung von Heiltees. Die gesammelten Teemengen mussten für ein Jahr Beratungstätigkeit reichen; dann ersetzte sie die Mischungen durch eine frische Ernte und verordnete die allenfalls übrig gebliebenen vorjährigen Kräuter als Handund Fussbäder oder auch als Inhalationen, z.B. Tannensprossenaufgüsse gegen Katarrh.

Jahreszeitengemäss sammelte sie zudem in anderen naturbelassenen wie auch alpinen Gegenden wie etwa dem Glarner Kurort Braunwald, dem graubündnerischen Davos oder in den Seitentälern des Wallis. Mit ansteckender Begeisterung gab sie dort ihr naturheilkundliches Wissen an Kursteilnehmerinnen und Kursteilnehmer weiter. Am liebsten unterrichtete sie unter freiem Himmel auf Kräuterwanderungen, wo sie immer auch auf den respektvollen Umgang mit der Natur aufmerksam machte (Abb. 3).

\section{Qualitätsaspekte handverarbeiteter Heilmittel}

Schwester Paulines jährlich selbst zubereitete Heilmittel waren bei ihren Patientinnen und Patienten beliebt und dienten zur pflanzenheilkundlichen Grundtherapie, welche sie individuell für einen kleinen Unkostenbeitrag verabreichte. Immer wieder konnte Schwester Pauline beobachten, dass ihre Kundschaft zurückkam, um Heilmittel von ihr zu beziehen, auch wenn die Ratsuchenden das von ihr verschriebene Rezept in einer näher gelegenen Apotheke oder Drogerie hätten mischen lassen können.

Schwester Paulines Kräutermedizin hatte bei ihrer Kundschaft die Reputation, von besonders guter Qualität und therapeutisch wirksam zu sein. In Vorträgen und im Kursunterricht legte sie grossen Wert darauf zu betonen, dass Pflanzen, die zu jahreszeitlich korrekten Zeiten an naturbelassenen Orten wild gesammelt werden, besonders heilkräftig sind [1]. Blattkräuter erntete sie in der Hauptwachstumsphase, Blüten zur Hauptblütezeit, Samen je nach Reifezeit im Spätsommer oder Herbst und Wurzeln im späten Herbst oder kurz vor Frühlingsanfang. Sie war überzeugt, dass diese traditionelle Sammelweise einen hohen Gehalt der heilenden Inhaltsstoffe sicherstellte.

Gewappnet mit guten botanischen Kenntnissen sammelte sie die gewünschten Kräuter nachhaltig von Hand und verarbeitete sie schonend zu traditionellen Heilmit-

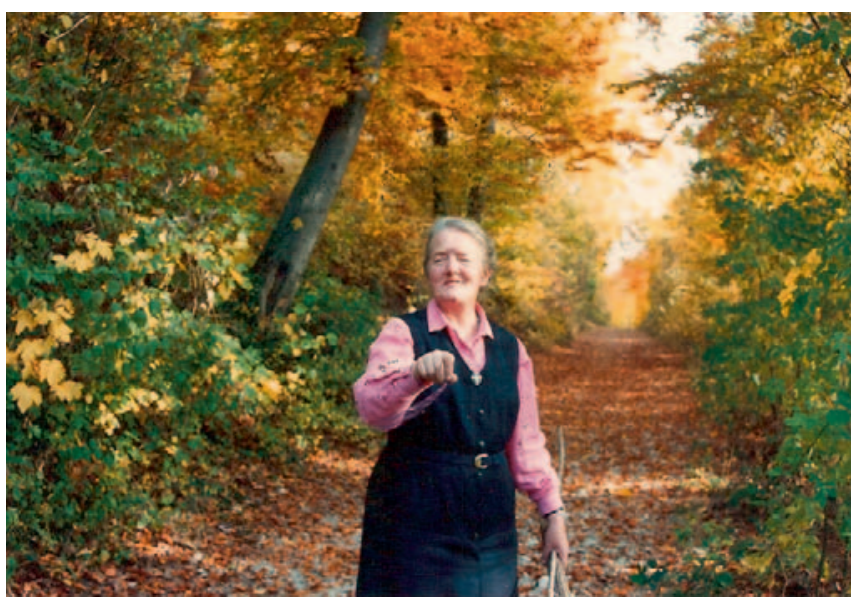

Abb. 3. Schwester Pauline auf einer Kräuterexkursion im Jahr 1989. In der freien Natur fühlte sie sich inspiriert zum nachhaltigen Sammeln von Heilpflanzen, die sie vorwiegend frisch zu traditionellen Heilmitteln verarbeitete (Copyright: Seraphisches Liebeswerk Solothurn).

teln. Im Gegensatz zu industriell hergestellten Heiltees unterwarf Schwester Pauline die ihrigen keinerlei chemischen oder thermischen Prozessen. So konnte es vorkommen, dass es in der Lagerung von getrockneten Pflanzen manchmal zu einem Vorkommen von pflanzenfressenden (aber sonst harmlosen) Motten kam. Trotz dieser manchmal vorhandenen unerwünschten Zusätze war es gemäss der Beobachtung der Erstautorin ein typischer Verlauf eines Beratungsgesprächs, dass die Patientinnen und Patienten auf die Heilwirkung von Schwester Paulines Kräutermedizin hinwiesen und eine Rezeptwiederholung erbaten. Eine frische und minimale Verarbeitung von Pflanzenmaterial trug in der Sicht von Schwester Pauline wesentlich zur Wirkung ihrer Mittel bei.

Traditionellerweise wurde neben den überlieferten technischen Regeln zur Herstellung von Pflanzenheilmitteln zusätzlich der geistigen Haltung während der Verarbeitung Bedeutung zugemessen - ein Aspekt, der in der industriell hergestellten, modernen Verarbeitung von Phytopharmaka kaum berücksichtigt wird. Schwester Pauline bereitete ihre Naturheilmittel mit dem Gedanken zu, ihrer Kundschaft zu helfen, und besang die entstehenden Präparate mit einem fröhlichen Herzen und einer Fürbitte für die Heilung der Ratsuchenden. Sie war davon überzeugt, dass diese Art der bewussten Verarbeitung die Heilkraft der Mittel verstärken und so auch «über die Seele» helfen würde [5].

Es ist zu vermerken, dass eine solche Miteinbeziehung einer Fürbitte bei der Heilmittelzubereitung in alten europäischen Arzneibüchern verzeichnet ist und somit eine Fortsetzung eines alten Brauchs darstellt. Noch bis in das 18. Jahrhundert stand die europäische Medizin unter dem Einfluss einer christlich-religiös fundierten Kosmologie mit dem expliziten und impliziten Postulat, dass Gottes Wirken in der Natur auffindbar und der Mensch ein Teil 
deren sei. Erst die Durchsetzung der wissenschaftlichen Revolution infolge der Aufklärung und die damit einhergehende Säkularisierung der Wissenschaft ersetzte diese Interpretation mit einem alternativen Deutungsmuster, das zur profanen Auffindung und Reproduktion von angenommenen Naturgesetzen aufrief [6]. Damit erst fand eine zunehmende kartesianische Trennung von Natur und Mensch statt - ein dualistisches Denken, das auch die Medizin grundlegend beeinflusste.

Das althergebrachte medizinische Naturverständnis ist am sichtbarsten in der europäischen Kulturgeschichte und Folklore, welche mittels mündlicher Transmission bis in die Gegenwart weitergegeben wurden [7]. Heutige volksmedizinische Sammelrituale legen Wert auf den respektvollen Umgang mit der Natur und die sorgfältige Ernte und Verarbeitung von Heilpflanzen, die damit besonders wirksam werden sollen.

Hier zeigt sich exemplarisch das Aufeinanderprallen von zwei verschiedenen medizinischen Paradigmen [8]. Der moderne Ansatz fokussiert auf die materielle Anwesenheit von einzelnen Inhaltsstoffen der Pflanze, während die traditionelle, hippokratische Naturphilosophie als Merkmal und Grundlage alles Lebendigen eine universale Lebensenergie annimmt [9]. Diese soll zusätzlich zu den materiell vorhandenen Inhaltsstoffen einer Pflanze und diesbezüglichen spezifischen pharmakologischen Wirkungen die allgemeine menschliche Konstitution und Vitalität stärken.

\section{Schritte zu einer erweiterten Naturheilpraxis}

Angetrieben von dem Bedürfnis, ihren Mitmenschen zu helfen, besuchte Schwester Pauline naturheilkundliche Fortbildungen, unter anderem beim Kneipp-Verein und beim Schweizerischen Verein für Natürliches Heilen (SVNH), sowie psychologische Weiterbildungen bei dem an der Universität Fribourg doktorierten Psychologen Dr. Beat Imhof, wie auch bei Maria Gasser und Katrin Giger.

In dieser Zeit las sie alles, was sie zum Thema «natürliches Heilen» finden konnte. Ein Heilpflanzenbuch von Professor Wagner, Direktor des Instituts für pharmazeutische Arzneimittellehre der Universität München, und ein praktischer heilpflanzlicher Ratgeber von Kaplan Bernhard Weber bestärkten ihren Wunsch, die Kräuterheilkunde weiter zu vertiefen [1]. Die Bücher von Maurice Mességué gaben ihr den Anstoss, Fuss- und Handbäder mit spezifischen Heilpflanzen auszuprobieren. Dass die Kinder in Bombinasco nach einem solchen abendlichen Handbad immer ruhiger schliefen, ermutigte sie, mit dieser Methode fortzufahren [2, S. 58]. So kam diese zum Einsatz bei Erkältungen, Nieren- und Blasenproblemen oder Kopfschmerzen. In ihrer späteren Gesundheitsberatungsstelle in Solothurn verliess kaum je eine Patientin

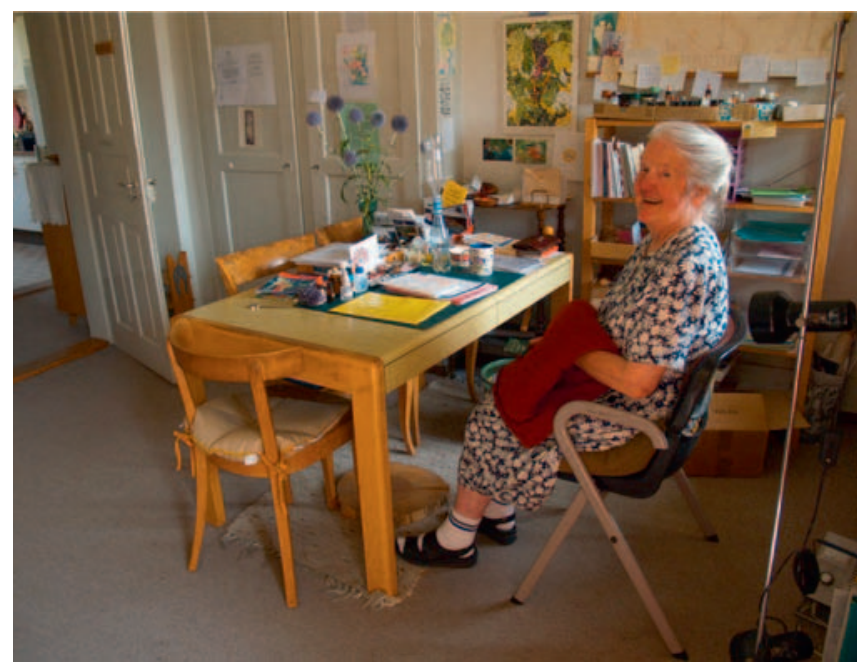

Abb. 4. Die Gesundheitsberatungsstelle am Nelkenweg 14 in Solothurn, in der die Klosterfrau von 1990-2007 mit ihrem Helferteam während ihrer bekanntesten Jahre um die 1800 Patientinnen und Patienten pro Jahr betreute (Copyright: Sandra Clair).

oder ein Patient ihre Praxis ohne eine Kräutermischung für eine diesbezügliche Hydrotherapie (Abb. 4).

Nahrung verstand Schwester Pauline als Heilfaktor. Ihre Überzeugung, dass Gesundheit am besten mit vollwertigen, unraffinierten und naturbelassenen biologischen Nahrungsmitteln erhalten bleibt, stammte aus Beobachtungen der frugalen Ernährung während ihrer Kindheit [1, S. 14]. Sie befasste sich zunehmend mit den Ernährungsprinzipen der Ärzte Bircher-Benner [10, 11] und Jarvis [12], dem österreichischen Naturheilpraktiker Breuss [13] und dem Naturarzt Alfred Vogel [14, 15]. Sie selbst wurde zur überzeugten Vegetarierin und wies darauf hin, dass schon der Schweizer Kräuterpfarrer Johann Künzle (1857-1945) den zu grossen Fleischkonsum kritisierte [16]. In ihren Konsultationen ging es ihr aber vor allem darum, die Eigenverantwortung zum massvollen Umgang mit Fleisch anzuregen, statt ihre Patientinnen und Patienten zum Vegetarismus zu überreden.

Schwester Pauline bildete sich auch in Bezug auf die Bausteine des Lebens weiter und befasste sich mit der Rolle von Vitaminen, Mineralien und Spurenelementen für den menschlichen Organismus. Bis ins hohe Alter war sie in der Lage, auswendig aufzuzählen, welche Nahrungsmittel reich an bestimmten Inhaltsstoffen sind, die spezifische Beschwerden positiv beeinflussen können. Sie selbst kochte liebend gerne und wertete ihre Mahlzeiten, wann immer möglich, mit frischem Wildgemüse und Wildfrüchten auf. Die Inspiration dazu entnahm sie verschiedenen Feld-, Wald- und Wiesenkochbüchern [17-19].

Volksmedizinische Bücher von diversen Autoren wie Pfarrer Kneipp [20, 21], Pfarrer Künzle [16], Alfred Vogel [14] und später Maria Treben [22, 23] und Bruno Vonarburg [24], welche tradiertes phytotherapeutisches Wissen 
dokumentieren, hatten einen festen Standplatz in Schwester Paulines naturheilkundlicher Bibliothek. «Das grosse Kräuterbuch» von Pfarrer Künzle (1857-1945), dem Kräuterpfarrer aus Zizers, nahm eine Sonderstellung ein. Der Pionier der Schweizer Pflanzenheilkunde basierte sein Buch im Wesentlichen auf der «Materia Medica» des Renaissancearztes Tabernaemontanus, dessen umfassendste deutschsprachige Heilpflanzenenzyklopädie im deutschsprachigen Kulturraum bis in das 18. Jahrhundert ein Standardwerk der akademisch-medizinischen Ausbildung war [25, 26]. In den 1970er-Jahren wurde Künzles Kräuterbuch von dem Nestor der modernen Phytotherapie, Dr. med. R.F. Weiss, und weiteren Ärzten unter modernen Gesichtspunkten durchgesehen und ergänzt. Dieses beliebte Werk trug somit dazu bei, dass erprobtes Heilpflanzenwissen der Renaissance bis in unsere Zeit weitertradiert und angewendet wird.

Schwester Pauline wurde ordentliches Mitglied des Kneipp-Vereins und liess fortan bei keiner Wanderung die Gelegenheit aus, ihre Arme oder Beine mit Kaltwasser zu therapieren. Die von Kneipp-Ärzten verbreitete Gesundheitstheorie der fünf Säulen Wasser, Ernährung, Bewegungstherapie, Heilkräuter und ordnende Pflege des Gemüts integrierte sie nun in ihre therapeutischen Empfehlungen. In Anlehnung an ihr Verständnis von Kneipps Lehrsätzen war sie der Überzeugung, dass kleine Reize die Gesundheit fördern, während allzu starke das Gegenteil bewirken sollen [1, S. 31].

Das Interesse der Klosterfrau galt allem, was die Natur hervorbringt, allem, was lebt, atmet und schwingt, und allem, was der Schöpfer zur natürlichen Heilung zur Verfügung stellt [3]. Sie trat dem SVNH bei, der sie später zum Ehrenmitglied ernannte. Sie beobachtete, las und experimentierte und war dabei in eklektizistischer Weise offen für unorthodoxe Heilmethoden, die in den 1980erJahren einen Aufschwung erlebten - unter der Voraussetzung, dass sie praktisch anwendbar waren und eine für sie überprüfbare positive Wirkung zeigten.

So erweiterte sie das Repertoire ihres beträchtlichen phytotherapeutischen Wissens durch Anwendungen mit Schüssler-Salzen, Bachblüten und Farbtherapie. Neben der pflanzlichen Basistherapie setzte sie auch die Baumheilkunde $[27,28]$ und die Edelsteintherapie nach der heiligen Hildegard ein - nicht nur wegen der zugeschriebenen Wirkung, sondern auch, um ihren Patientinnen und Patienten ein anfassbares Hilfsmittel in die Hand zu geben und ihre Gedanken aktiv auf Heilung auszurichten, sozusagen als Heilmeditation. Somit hatten die Edelsteine und Baumzweige, welche die Patientinnen und Patienten täglich zu halten hatten, durchaus rituellen Charakter und sollten ihnen auch dazu dienen, sich auf die Heilkräfte der Natur zu besinnen.

Es war ein Buchgeschenk ihres Cousins zur Edelsteinkunde der heiligen Hildegard [29], das den Auftakt zu ihrer intensiven Beschäftigung mit der heiliggesprochenen Äbtissin aus dem 12. Jahrhundert gab, zu der sie eine Affinität spürte und die fortan einen wichtigen Platz in ihrem Leben einnahm. Ihr Interesse an Traditioneller Chinesischer Medizin (TCM) und Ayurvedischer Medizin führte sie zu feinstofflichen körperregulierenden Systemen wie Meridianen und Chakren.

In dieser Zeit nahm sie auch rege an Meditationskursen über kreative Visualisierung und Geistheilung teil und integrierte diese Art geistige Übung in ihre religiösen Praktiken. Mit täglichen Meditationen schulte sie ihre Wahrnehmungs- und Beobachtungsgabe und damit auch ihre «innere Stimme» [2, S. 127].

Als Ordensschwester war sie überzeugt von der Heilkraft positiver Gedanken, hatte sie als Nonne doch tägliche Erfahrung mit der aufbauenden Wirkung von strukturierten positiven Gedanken in Form von Gebeten. Nie liess sie eine Ratsuchende weggehen, ohne ein aufmunterndes Wort, Hoffnung und (Gott-)Vertrauen mit auf den Weg zu geben. Oft legte sie auch die Hand auf eine kranke Stelle, während sie ihr liebstes Christusbild mit den innerlich gesprochenen Worten ansah: «Ich bin parat, jetzt übernimm Du.» Sie fühlte einen direkten Draht zur göttlichen Allmacht und hatte die absolute Gewissheit, dass eine solche spirituelle Fürbitte sowohl das körperliche als auch das seelische Wohlergehen ihrer Patientinnen und Patienten positiv beeinflussen konnte. Schwester Pauline verstand sich als Werkzeug Gottes, glaubte mit dem Herzen und strahlte dadurch ein spürbares Charisma aus, das seinerseits den Glauben und die Zuversicht in eine Heilung bei ihrer Kundschaft stärkte. Sie hatte ein unerschütterliches Vertrauen in die menschliche Kraft zur Genesung, welche sie mit natürlichen Mitteln anregen wollte.

Ihr Behandlungsmantra war ein mit Überzeugung gesprochenes «Wir wollen's versuchen», in dem ein klares «Alles ist möglich» mitschwang. Das echte Mittragen der Nöte und Sorgen der Ratsuchenden zusammen mit ihrer vertrauenswürdigen Persönlichkeit führten zu einer positiven psychosozialen Intervention und gaben, neben dem Verschreiben einer Individualrezeptur, einen nicht zu unterschätzenden Heilimpuls. In welcher Situation auch immer sich ihre Patientinnen und Patienten befanden, sie fühlten sich bei ihr in erster Linie bestmöglich aufgehoben. Ein wichtiger Teil ihrer Arbeit als Ordensfrau war auch die Stärkung des Selbstwertgefühls und in gewisser Hinsicht emanzipatorische Aufklärung bei Frauen aus konservativen katholischen Familien und Gegenden, denen sie Mut zu einem eigenständigeren Leben machte. Mit ihrem menschlichen Engagement, echter Anteilnahme, guten Menschenkenntnissen und ansteckendem Humor zeigte sie eine spezielle Begabung und war in der Lage, eine psychosoziale Lücke zu füllen, die viele ihrer Patientinnen und Patienten nach einer anderswo erfolgten schulmedizinischen Behandlung zu empfinden schienen. 
Parallel zu ihrer naturheilkundlichen Tätigkeit vertiefte sich auch ihre Spiritualität, welche sich letztlich von ihrer Heiltätigkeit nicht trennen lässt. So fühlte sie sich der Mystik des Johannes-Evangeliums immer näher und beschäftigte sich des Weiteren mit Schweizer Heiligen wie dem Einsiedler Bruder Klaus, in dessen Kapelle an seinem Geburtsort Braunwald sie gerne innere Einkehr nahm. Der Kontakt mit der «geistigen Welt» wurde ihr zur Selbstverständlichkeit, ohne dabei den Boden unter den Füssen zu verlieren [2]. Ihre zunehmende Offenheit und Neugierde führten jedoch auch zu brennenden spirituellen Fragen betreffend der tieferen Bedeutung evangelischer und spiritueller Texte, d.h. Fragen, welche sie im Ordenskreis nicht ansprechen konnte und die sie deshalb lieber mit ausserklösterlichen Freunden wie dem Mathematiker, Philosophen und Lebensberater Dr. Alexander Gosztonyi diskutierte [30].

Mit zunehmender Sensibilität in den 1990er-Jahren empfand sie geopathologische Wasseradern und den modern auftretenden Elektrosmog als gesundheitsbedrohliche Störzonen, vor denen sie warnte. Dass schnurlose Telefone in Abhängigkeit von Strahlungsstärke und Entfernung die Hirnströme verändern, ist heute aus der Hirnforschung belegt. Inwiefern diese neben Kopfschmerzen und Ohrensausen auch bleibende Schäden verursachen, ist sowohl von akademischem als auch behördlichem Interesse [31,32]. Für Schwester Pauline war der Sachverhalt klar, erlebte sie doch immer wieder ein spontanes Versagen ihrer Stimme, wenn sie solchen Strahlenquellen ausgesetzt war [33].

\section{Gesundheitsberatungsstelle Solothurn}

Schwester Paulines Ruf als heilkundige Klosterfrau hatte zur Folge, dass sich immer mehr Hilfesuchende bei ihr meldeten. Ihre naturheilkundliche Beratungstätigkeit wurde nun zum zentralen Fokus ihres Tagesablaufs. $\mathrm{Zu}$ nächst empfing die Ordensfrau die Ratsuchenden im Kloster. Doch dort wurde es bald zu eng. In unmittelbarer klösterlicher Nähe, an der Werkhofstrasse 17, fand sie ein Büro, welches sie zusammen mit Assistentinnen und Assistenten als Gesundheitsberatungsstelle einrichtete. Ein Bericht in der Zeitschrift Beobachter über die Heilerfolge der religiösen Kräuterfrau löste eine Lawine von Briefen (gut 20 pro Tag), Telefonanrufen (50-70 pro Tag) und persönlichen Besuchen aus [2, S. 76].

Bald kamen weitere Zeitungsinterviews, Vorträge, Seminare, Kräuterwanderungen und Fernsehauftritte dazu, sodass dieses Büro für den zunehmenden Besucherandrang bald ebenfalls zu klein war. Nach einem Intermezzo im Wallis während des Sommers 1990 fand Schwester Pauline in einem ruhigen Solothurner Wohnquartier eine grosszügig ausgelegte Wohnung, die sie als Gesundheits- beratungsstelle bis zu ihrem plötzlichen Hinscheiden mit 82 Jahren infolge von Komplikationen nach einer Hüftoperation nutzte. Am Nelkenweg 14 betreute sie während ihrer bekanntesten Jahre um die 1800 Personen pro Jahr und beantwortete, zusammen mit ihrem Helferteam, vielzählige persönliche, telefonische und schriftliche Anfragen $[1$, S. 10].

Offen sein, verstehen statt urteilen, da sein - dies war die gelebte Grundhaltung, mit welcher Schwester Pauline ohne Unterschied alle Hilfesuchenden empfing. Ihre Oberin vermerkte in ihrem Nachruf: «Durch ihre Kontaktfähigkeit, ihre Ausstrahlung und ihre Unkompliziertheit fand sie rasch den Zugang zu verschiedensten Menschen. Ihre Stärke war es, den Menschen ernst zu nehmen, zu respektieren unabhängig von seiner Intelligenz, seinem Stand oder seiner Herkunft» [3].

Die Ordensschwester war der tiefen Überzeugung, dass in der Natur für jede Krankheit ein Heilmittel zu finden ist. Keineswegs nahm sie jedoch in Anspruch, alles zu wissen, und war auch der Schulmedizin gegenüber offen, wenn sie keine begründbare Alternative kannte [34]. Im Zentrum standen Hilfe zur Selbsthilfe und die überzeugende Ermunterung, eigene Erfahrungen zu sammeln, um herauszufinden, was zur persönlichen Gesundung beiträgt. So betonte sie immer wieder, dass jeder an seiner Genesung mitarbeiten muss und diese nicht an den Arzt oder Heilpraktiker abdelegieren kann [1]. In ihren Therapievorschlägen ging es darum, die körpereigene Heilkraft anzuregen und erschöpfte Reserven wieder aufzubauen. Ihre Vorgehensweise steht im Kontext einer ganzheitlichen Heilkunde, die unter Berücksichtigung der individuellen Konstitution und Lebensführung Homöostase bewirken will [35].

Viele ihrer Patienten suchten die Gesundheitsberatungsstelle wegen chronischer Beschwerden, Schmerzen und funktioneller Störungen auf, wie persönliche Beobachtungen der Erstautorin sowie einige hinterlassene rudimentäre Berichte von Patientinnen und Patienten bestätigen [2, S. 132-142]. Derartige Beschwerden sind sozialmedizinisch äusserst bedeutsam, weisen jedoch schulmedizinisch nur einen bedingten und oft mit $\mathrm{Ne}$ benwirkungen belasteten Behandlungserfolg auf. Die Traditionelle Europäische Medizin (TEM) offeriert hierzu über lange Zeiträume erprobte und in zunehmendem Mass auch durch moderne Studien belegte pflanzliche Heilmittel. Schwester Pauline setzte solch altbewährte Heilpflanzen in ihren Konsultationen gezielt und mit Erfolg ein. Zusätzlich war es ihr ein Anliegen, ihren Patientinnen und Patienten mit zum Teil unkonventionellen Hilfsmitteln die Hoffnung und den Willen zum Gesundwerden zu stärken.

Schwester Paulines Aktivitäten blieben ihr Leben lang streng strukturiert und folgten einem geordneten Tagesund Wochenablauf. Auch Spass durfte in diesem Rhyth- 
mus nicht fehlen, und so freute sie sich immer auf den Sonntags-Jass mit ihren Mitschwestern [4]. Selbst wenn ihre starke Persönlichkeit gelegentlich zu lebhaften Diskussionen mit anderen Klosterfrauen führte und sie durch ihre Heiltätigkeit in ihrem letzten Lebensdrittel einen Grossteil ihres Tages ausserhalb der Klostermauern verbrachte, fühlte sie sich stark mit dem SLS verbunden. Die Geborgenheit und Sicherheit der religiösen Gemeinschaft mit den täglichen gemeinsamen spirituellen Praktiken gaben ihr einen ruhenden Anker.

\section{Das Pendel: Diagnose- und Rezepturfindungsinstrument}

Gestützt auf ihre breite praktische Erfahrung verschrieb Schwester Pauline für jeden Ratsuchenden eine passende Individualrezeptur. Als Kontrolle und als Unterstützung bei der Anamnese verwendete sie zudem ein Pendel, denn eine familiäre Eigenheit sollte sich zu einem unorthodoxen, aber zentralen Werkzeug von Schwester Paulines Arbeit entpuppen. Sowohl ihr Grossvater als auch ihr Onkel waren für ihre aussergewöhnliche Begabung bekannt, in ländlichen Gegenden mithilfe des Pendels Wasser zu finden. Diese Pendel-Gabe entdeckte sie in ihren frühen Dreissigern beinahe zufällig aufgrund eines Notfalls auch bei sich, als sie bei der Suche nach einem Vermissten dessen Aufenthaltsort per Pendel bestimmen konnte. Später, als junge Hebamme im Mütternheim Belfond, welches nur rudimentär und ohne Ultraschall ausgestattet war, pendelte sie auf Anfrage der werdenden Mütter das Geschlecht der ungeborenen Kinder. Doch erst als Mittfünfzigerin war sie bereit, ihre Pendelkraft gezielt auch in Gesundheitsfragen als Diagnose- und Rezepturfindungsinstrument einzusetzen.

Neben der Anamnese untersuchte sie auf diese unorthodoxe Art verschiedene Körpersysteme auf Störungen, spürte Defizite auf und erzielte manchmal auch sensationelle Funde, wie etwa ein während einer Operation im Körper vergessenes chirurgisches Instrument, das einer Patientin trotz Spezialistenbetreuung über Jahre hinweg unerklärliche Beschwerden verursacht hatte [36]. Solche Berichte breiteten sich wie ein Lauffeuer aus, sodass Schwester Paulines Terminkalender oft über Wochen ausgebucht war.

Die Verwendung von Divinationsinstrumenten ist uralt. In Europa wird die Radiästhesie seit dem 19. Jahrhundert in der wissenschaftlichen Literatur beschrieben, fand jedoch keinen Zugang in die moderne Medizin und zählt heute zum Bereich der randständigen Parawissenschaften. Dessen ungeachtet war Schwester Paulines Pendelgabe bei ihrer Klientel beliebt und wurde von vielen als integraler Bestandteil ihrer Diagnostik geschätzt. Hier beispielhaft eine Anekdote aus einem Forum: «Ich war selber
13 Jahre kinderlos. Pauline Felder, eine Klosterfrau, die vor kurzem gestorben ist, hat für mich Verschiedenes ausgependelt. Mein Schlafplatz war geopathisch belastet. Ich habe eine Bachblütenmischung genommen. Mein Mann und ich haben täglich eine Tasse Storchenschnabeltee getrunken. Nach sehr kurzer Zeit bin ich dann schwanger geworden. Béatrice» [37].

Schwester Pauline stiess mit ihrer Pendelgabe aber auch auf Misstrauen und sorgte für Kritik. Der Beitrag heilkundiger Ordensfrauen zur Gesundheitsversorgung der Bevölkerung ist in Europa zwar ein jahrtausendealter Teil klösterlicher Tradition [38-40]; so inspirierte der Karolingische Klosterplan der Stiftsbibliothek St. Gallen aus der Zeit um 820 mit seinem Kräutergarten und medizinischen Bezirk die europäische Klostermedizin. Doch das Pendel als Arbeitsinstrument war einem besuchenden Geistlichen aus Rom wie auch einigen ihrer Mitschwestern suspekt. Sie befürchteten, dass das Pendeln im besten Fall mit Aberglaube, im schlimmsten Fall aber mit Magie zu tun habe. Zwar wurde es in der katholischen Kirche gebilligt, wenn Geistliche auf ihren Missionsstationen Wasser auspendelten, doch der wiederholt vom Vatikan entsandte Pater Gambari war mit Schwester Paulines Pendeln in Gesundheitsfragen zunächst nicht einverstanden. Die Ordensschwester blieb allerdings standfest und berief sich auf Stellen in der Bibel, wie etwa Moses' Verwendung der Gabelrute. Nach langen Interviews konnte sie den Abgeordneten aus Rom überzeugen, dass ihre Gabe eine Gabe Gottes sei, die sie alleinig dafür einsetze, ihren Mitmenschen zu helfen. Schliesslich erhielt sie vom Vatikan die offizielle Erlaubnis, für Hilfesuchende weiterzupendeln $[1 ; 2$, S. 44, 76]. Dabei war sicherlich auch wichtig, dass ihr Bischof und ihre Oberin, welche sie über Jahrzehnte seelsorgerisch begleitet hatten, ihre persönliche Integrität bezeugten.

Für Schwester Pauline war das Pendeln sichtbar gemachte Intuition, die sie zur Bestätigung und Ergänzung ihres geschulten Wissens einsetzte. Sie war eine erfahrene Naturheilpraktikerin aufgrund ihres fachmedizinischen Wissens in der Geburtsheilkunde. Diese intuitiv erfassten Informationen verwendete sie gezielt neben der klinischen Beurteilung. Intuition, die auf Erfahrung beruht, ist von spontaner Intuition zu unterscheiden und ergänzt professionelle Expertise in einer patientenorientierten Betreuung [41]. Schwester Paulines Intuition kann als geschulte Intuition bezeichnet werden, die sich trotz der Berufung auf ein ungewöhnliches Hilfsmittel auf klinische Erfahrung abstützte.

\section{Psychosoziale Aspekte der Heilkunde}

Es wäre einfach, den psychosozialen Aspekt von Schwester Paulines Konsultationen als «Placebo» abzu- 
tun. Fortschritte in der Placeboforschung sowie qualitative Analysen wie «Whole Systems Research» [42] und «Comparative Effectiveness Research» [43] in der Komplementär- und Biomedizin weisen darauf hin, dass «Placebo» ein erwünschter und kalkulierbarer kontextueller Heileffekt ist. Die Vorgehensweise einer Therapeutin oder eines Therapeuten während einer Konsultation, die Überzeugungen und die menschlichen Werte beeinflussen entscheidend das Heilgeschehen [44, 45]. Der «Therapeut als Medikament» hat eine wesentliche und messbare Wirkung auf das Patientenverhalten und das Ergebnis einer Behandlung - ein Effekt, der über die medikamentöse oder physikalische Therapie hinausgeht [46, 47]. Sogar bei rein medikamentösen Behandlungen können Erwartungen und Einschätzungen der Patientinnen und Patienten bedeutsamer die Wirksamkeit beeinflussen als die geprüften pharmakologischen Effekte der Arzneimittel. Dies hat z.B. die Reanalyse einer placebokontrollierten klinischen Studie mit Sertralin und einem Johanniskrautextrakt exemplarisch gezeigt [48]. Komplementärmedizinische Beratungen erfreuen sich vielleicht gerade deshalb weltweit einer steigenden Beliebtheit, weil sie grossen Wert auf den Kontext einer Behandlung legen, welche Zuspruch, empathisches Zuhören und adäquate Länge einer Konsultation beinhaltet.

Dass Gedanken und Gefühle die körpereigenen Abwehrkräfte stimulieren können oder - mit anderen Worten - dass eine Wechselwirkung zwischen Psyche, Nervensystem und Immunsystem besteht, ist hinreichend belegt und wird beispielsweise in der Psychoneuroimmunologie und Psychoneuroendokrinologie untersucht.

\section{Pauline Felders schriftliches Vermächtnis}

Schwester Pauline war keine Akademikerin, sondern eine heilkundige Ordensfrau und Vertreterin der gelebten schweizerischen Volksheilkunde. Ihre Aufmerksamkeit galt dem praktischen Handeln und nicht einer methodischen Dokumentation ihrer Beobachtungen und Erfahrungen. Dies ist wohl auch ein Grund, weshalb Schwester Paulines schriftliche Erzeugnisse in vielen Bereichen aus dem kontextuellen Rahmen fallen und keine umfassende Reflektion ihrer Beratungstätigkeit repräsentieren, sondern eher eine kaleidoskopische Beleuchtung verschiedener Aspekte ihrer Interessen sind - von der TEM bis zu neueren Themen wie Elektrosmog oder feinstofflichen Energiesystemen, mit denen sie sich in ihrem letzten Lebensabschnitt befasste. Wer Schwester Paulines Arbeit begleitet hat, stellt fest, dass viele der von ihr angewendeten phytotherapeutischen Heilmittel in ihren Büchern nicht festgehalten sind, vielleicht weil diese für sie selbstverständlich waren. Aus diesem Grund blieb die Gesamtheit ihres Heilwissens nur unvollständig dokumentiert.
Ihr am besten bekanntes Buch ist der 1988 erschienene Ratgeber zur Erfahrungsheilkunde «Gesundheitsbrevier. Alternative Heilmethoden - praktisch erprobt» [1]. Es beinhaltet eine Überarbeitung ihrer erwähnten monatlichen Kolumne "Gesund bleiben - gesund werden» durch die Journalistin Jeanne Glutz-Weber. Dieses Buch wurde auch ins Französische übersetzt und mehrere Male aufgelegt [49]. 1992 folgten ihre Erfahrungen mit der Heilkraft von einheimischen Bäumen [28] und 1997 eine Zusammenstellung ihrer Vorträge [50]. Autobiographische Daten sind ihrem Büchlein «Wie er mich führte» zu entnehmen [2]. Ferner legte Schwester Pauline eine Broschüre von Kaplan Bernhard Weber neu auf, die sie seinerzeit anspornte, ihr Wissen über Kräuter zu vertiefen [51]. Ihr obstetrisches Wissen ist nicht schriftlich dokumentiert, doch verwies sie in ihrer Praxis des Öfteren auf das Buch «Die Hebammensprechstunde» ihrer Berufskollegin Ingeborg Stadelmann [52].

\section{Diskussion}

Es ist ein komplexes Unterfangen, eine Praxis zu beschreiben, deren Heilmittel wesentlich auf mündlichen und volksmedizinischen Überlieferungen basierten, in der gerade auch die psychosoziale Intervention eine so zentrale Rolle bei dem Erreichen der Heilerfolge spielte und die vom Glauben und Charisma der Heilpraktikerin getragen war. $\mathrm{Zu}$ einer vollständigen Analyse hätte es einer ethnographischen Beobachtungsstudie bedurft. «Rapid Ethnographic Observation» ist eine wissenschaftliche Methode, die auf Studien der medizinischen Soziologie basiert [53] und die mittels teilnehmender Beobachtung des Beratungsvorgangs sowie nachträglichen Interviews mit der/dem Therapeutin/Therapeut und der/dem Patientin/Patient zentrale Informationen zum Kontext sowie zur Patientenmotivation, Diagnose und Wirkung einer Behandlung liefert $[54,55]$. Quantitative Untersuchungsmethoden wie die randomisierten kontrollierten Doppelblindstudien, die für die Evaluation der Wirksamkeit eines Pharmakons entwickelt wurden, sind methodologisch nicht geeignet, qualitative Aspekte einer Behandlung zu evaluieren $[56,57]$. Eine ethnographische Studie hätte Aufschluss über die Hintergründe von Schwester Paulines Popularität gegeben, die Beweggründe der Patientinnen und Patienten, sie - anstelle von oder zusätzlich zu einer biomedizinischen Behandlung - aufzusuchen, was sich genau während der Konsultation abspielte und schliesslich eine Analyse der Wirksamkeit ihrer Methoden. Solche Studien sind wichtig, denn sie adressieren ein echtes Bedürfnis in der Bevölkerung nach traditionellen, komplementärmedizinischen und unorthodoxen Methoden [58]. Sie können offenlegen, wie traditionelle Heilerinnen und Heiler in ihren Konsultationen vorgehen, wel- 
che Heilmittel und Methoden sie einsetzen und welche therapeutischen Wirkungen sie bei spezifischen Beschwerdebildern erzielen. Solche Untersuchungen tragen zu einem besseren Verständnis einer patientenzentrierten Medizin bei, welche nicht nur auf naturwissenschaftlich orientierten klinischen Untersuchungen basiert, sondern auch Aspekte der klinischen Empirie beinhaltet [44, S. 160].

Historische Indikationsanalysen haben erwiesen, dass empirisch gewachsene Verordnungen vergangener Zeiten nicht a priori abgesprochen werden können und dass ein Zusammenhang zwischen Indikationsangaben von frühzeitlichen, mittelalterlichen und neuzeitlichen Autoren und modernen Anwendungen besteht [59-62]. Zentrale Heilpflanzen der TEM sind z.B. bereits in Dioskurides' Pharmakopöe «De Materia Medica» dokumentiert und weisen über eine Spanne von über 2000 Jahren eine erstaunliche Indikationskonsistenz auf [63]. Im Gegensatz zu dieser schriftlichen Tradition sind regionale, mündliche Transmissionen grösseren Instabilitäten unterworfen. Es wäre deshalb lohnenswert, gegenwärtiges volksmedizinisches Wissen der europäischen Erfahrungsheilkunde mittels ethnographischer Beobachtungsstudien zur Ethnobotanik, zur Ethnopharmakologie und zu ihrem Anwendungskontext aufzubereiten.

Auf internationaler Ebene erkennt die World Health Organisation (WHO) den Beitrag an, den die traditionelle Medizin zur Gesundheitsversorgung einer Bevölkerung liefern kann, und verfolgt mit ihrer «Traditional Medicine Strategy 2014-2023» eine Verbindung von traditionellen Medizinsystemen mit der Biomedizin [64, 65]. In der Schweiz wie auch in anderen Ländern Zentraleuropas sind im Zuge der Professionalisierung heilkundige Ordensleute wie Schwester Pauline und traditionelle Laienheilpraktikerinnen und -praktiker jedoch immer seltener anzutreffen. Daher besteht eine hohe Dringlichkeit, Ressourcen für die Dokumentation des mündlich tradierten europäischen Heilwissens wie auch für die gezielte Aufarbeitung historischer «Materia Medicae» verschiedener einflussreicher ärztlicher Autoren zur Verfügung zu stellen, um ein umfassenderes Bild der TEM zu erarbeiten.

Pflanzliche Arzneimittel repräsentierten bis in das 20 . Jahrhundert die Haupttherapeutika der europäischen Gesundheitsversorgung und wurden seit jeher auch zur Eigenbehandlung in der Bevölkerung angewandt. Der Miteinbezug der traditionellen lokalen Medizin einer Bevölkerung trägt $\mathrm{zu}$ dem von den Patienten geforderten medizinischen Pluralismus bei, der eine komplementäre Koexistenz von evidenz-, kognitions- und wertbasierten sowie empirischen Methoden zu einer ganzheitlichen Behandlung von Beschwerden berücksichtigt [66].

In ihrer phyto- und hydrotherapeutischen Arbeitsweise war Schwester Pauline eine originelle Vertreterin der schweizerischen Volksmedizin. Der langjährige Erfolg ihrer Solothurnischen Gesundheitsberatungsstelle zeigt exemplarisch, dass traditionell angewandte Heilmittel der europäischen Medizin bei den Patienten erwünscht sind. Auf dieser Basis erscheinen neben professionellem medizinischem Wissen auch psychosoziale Aspekte einer Behandlung von elementarer Bedeutung. Schwester Paulines Wirken und Vermächtnis dokumentieren, dass die schweizerische Volksmedizin eine lebende Tradition ist.

\section{Dank}

Ein besonderer Dank gilt Schwester Paulines engen Mitarbeiterinnen und Mitarbeitern der Solothurner Gesundheitsberatungsstelle, Hans-Ulrich Sieber und Christina Flury für reflektierende Gespräche, Christine Schwaninger Sieber für erweiterte Perspektiven, Schwester Marie-Theres Rotzetter, Oberin des SLS, für die Klärung von Fragen und Bildmaterial sowie Prof. Ian Coulter, University of California, Los Angeles (UCLA), USA, für die Besprechung der medizinsoziologischen Methode «Rapid Ethnographic Observation» zur Dokumentation von qualitativen Aspekten naturheilkundlicher Praxen.

\section{Disclosure Statement}

In Bezug auf diese Arbeit bestehen keine Interessenkonflikte.

\section{Literatur}

1 Felder P: Gesundheitsbrevier. Alternative Heilmethoden - praktisch erprobt. Arlesheim, Yerbasana, 1988.

2 Felder P: Wie er mich führte ... Arlesheim, Yerbasana, 1993.

3 Rotzetter MT: Zum Gedenken an Pauline Felder. Schwesterngemeinschaft Seraphisches Liebeswerk Solothurn. Geboren am 16. Januar 1925 - Gestorben am 20. Juli 2007. Nachruf. Solothurn, SLS, 2007.

4 Weiss C: Pauline Felder, 75, Naturheilerin. Modeblatt 2000;11:74

5 Felder P: Kräuter, die uns helfen gesund $\mathrm{zu}$ bleiben und gesund zu werden. Solothurn, Kursunterlage. Kein Datum.
6 Winnerling T: Kein Usus Medicus oder: Wie der Knoblauch seine Kräfte verlor. Köln, Böhlau, 2013, p 97.

7 Storl W-D: Naturrituale. Baden, AT, 2004.

8 Evans S: Changing the knowledge base in Western herbal medicine. Soc Sci Med 2008; 67:2098-2106.

9 Schmitz R: Geschichte der Pharmazie. 1. Von den Anfängen bis zum Ausgang des Mittelalters. Eschborn, Govi, 1998, p 115.

10 Bircher-Benner M: Ordnungsgesetze des Lebens. Drei Vorträge für die «Food Education Society». Bad Homburg (vormals Zürich), Bircher-Benner, 1992. (Nachdruck von: Zürich/Leipzig/Wien, Wendepunkt, 1938.)
Melzer J, Melchart D, Saller R: Entwicklung der Ordnungstherapie durch Bircher-Benner in der Naturheilkunde im 20. Jahrhundert. Forsch Komplementärmed Klass Naturheilkd 2004;11:293-303.

12 Jarvis DC: $5 \times 20$ Jahre leben. Bern/Stuttgart, Hallwag, 1985.

13 Breuss R: Krebs. Leukämie und andere scheinbar unheilbare Krankheiten mit natürlichen Mitteln heilbar. Wangen/Allgäu, Merk, 1978.

14 Vogel A: Der kleine Doktor. Teufen, A. Vogel, 1978.

15 Melzer J, Saller R: Der «Naturarzt» Alfred Vogel (1902-1996). Schweiz Zschr Ganzheitsmedizin 2003;15:66-72, 118-123. 
16 Künzle J: Das Große Kräuterheilbuch. Ratgeber für gesunde und kranke Tage. Durchgesehen und ergänzt von Dr. med. R.F. Weiss. Olten/Freiburg, Walter, 1974.

17 Heiss E: Wildgemüse und Wildfrüchte. Eine wertvolle Ergänzung und Aufwertung unserer heutigen Nahrung. München, Dr. Johann Herp, 1980.

18 Helm EM: Feld-, Wald- und Wiesenkochbuch. Erkennen, Sammeln, Zubereiten und Einkochen von Wildgemüsen und Wildfrüchten. Frankfurt am Main/Wien/Zürich, Büchergilde Gutenberg, 1978.

19 Quinche R, Bossard E: Wildfrüchte. Thun, Ott, 1973.

20 Kneipp S: Meine Wasser-Kur. Kempten, Kösels, 1901.

21 Kaiser JH (Hrsg): Das große Kneippbuch. Handbuch der naturgemäßen Lebens- und Heilweise. München, Ehrenwirth, 1975, p 10.

22 Treben M: Gesundheit aus der Apotheke Gottes. Ratschläge und Erfahrungen mit Heilkräutern. Karlstein/Thaya, Verein Freunde der Heilkräuter, ohne Datum.

23 Treben M: Maria Treben's Heilerfolge. Briefe und Berichte von Heilerfolgen mit dem Kräuterbuch «Gesundheit aus der Apotheke Gottes». Steyr, Wilhelm Ennsthaler, 1985.

24 Vonarburg B: Natürlich Gesund mit Heilpflanzen. Aarau, AT, 1993

25 Clair S: Die Kräutermedizin des RenaissanceArztes Tabernaemontanus (16. Jh.) und Phytotherapie heute - was ist geblieben, was hat sich verändert? Schweiz Z Ganzheitsmed 2011;23:46-52.

26 Kräuterpfarrer Künzles Gesundheitskalender. Das Jahrbuch der Gesundheit. Minusio-Locarno, Kräuterpfarrer Künzle AG, 1972.

27 Strassmann R: Baumheilkunde. Wilen, Renatus, 1983.

28 Felder P: Von der Heilkraft unserer Bäume. Arlesheim, Yerbasana, 1992

29 Hertzka G, Wighard S: Die Edelsteinmedizin der Hl. Hildegard. Freiburg i.Br., Hermann Bauer, 1985.

30 Gosztonyi A: Das Vaterunser: Die Entwicklung des Menschen im Lichte des Evangeliums - vor dem Hintergrund der Reinkarnation. Oberstdorf, Windpferd, 2002.

31 Kuster N: Abschätzung der durch Mobiltelefone (GSM, UMTS) induzierten niederfrequenten Ströme im menschlichen Kopf. Zürich, ETH, 2011. www.mobile-research.ethz. ch/fileadmin/redaktion/public/downloads/2_ foerderung/dokumente_projekte/Kuster_Abstract_de.pdf (aufgerufen am 24.03.2013).

32 Forschungsstiftung Mobilkommunikation. Liste der geförderten Forschungsprojekte. www.mobile-research.ethz.ch/de/foerderung/ projekte/liste-der-gefoerderten-forschungs projekte/\#refno-39 (aufgerufen am 24.02.2013).

33 Felder P: Achtung vor schädlichen Strahlen Computer, Fernseher, Handys, Umsetzer für Antennen usw. Solothurn, Merkblatt, kein Datum.

34 Gradmann M: Wenn Frau will, steht alles still! Schweizer Gastronomie 2000;3:21.
35 Kenner D, Requena Y: Botanical Medicine. A European Professional Perspective. Brookline, Paradigm, 2001.

36 Augenzeugenbericht der Erstautorin.

37 www.symptome.ch/vbboard/gesundheitfrauen/18625-unerfuellter-kinderwunsch.htm - 12.03.2008, 15:45 \#4 AW: Unerfüllter Kin derwunsch4 (aufgerufen am 24.02.2013).

38 Achterberg J: Die Frau als Heilerin. Die schöpferische Rolle der heilkundigen Frau in Geschichte und Gegenwart. Bern, Scherz, 1990

39 Mayer JG: Das geheime Heilwissen der Klosterfrauen. Reinbek bei Hamburg, Rowohlt, 2008.

40 Schmuki K, Tremp E, Otto N, Schumacher H Heilkräuter und Gartenanlagen im Kloster St. Gallen. Katalog zur Jahresausstellung in der Stiftsbibliothek St. Gallen (30. Novembe 2009-7. November 2010). St. Gallen, Verlag am Klosterhof, 2010.

41 McCarthy J, Alexander P, Baldwin M, Woodhouse J: Valuing professional judgment; in McCarthy J, Rose P (Hrsg): Value-Based Health and Social Care. Beyond EvidenceBased Practice. Los Angeles, Sage, 2010, pp 107-113.

42 Jonas WB, Beckner W, Coulter ID: Proposal for an integrated evaluation model for the study of whole systems health care in cancer. Integr Cancer Ther 2006;5:315-319.

43 Sox HC, Greenfield S: Comparative effectiveness research: a report from the Institute of Medicine. Ann Intern Med 2009;151:203205.

44 McCarthy, J, Rose, P (Hrsg): Value-Based Health and Social Care. Beyond EvidenceBased Practice. Los Angeles, Sage, 2010.

45 IOM: Complementary and Alternative Medicine (CAM) in the United States. Washington DC, National Academies Press, 2005.

46 Beauregard M: Brain Wars: The Scientific Battle Over the Existence of the Mind and the Proof that Will Change the Way We Live Our Lives. New York, HarperCollins, 2012.

47 Khan A, Khan S: Placebo in mood disorders the tail that wags the dog. Curr Opin Psychiatry 2002;16:35-39.

48 Chen JA, Papakostas GI, Youn SJ, Baer L, Clain AJ, Fava M, Mischoulon D: Association between patient beliefs regarding assigned treatment and clinical response: reanalysis of data from the Hypericum Depression Trial Study Group. J Clin Psychiatry 2011;72:1669_ 1676.

49 Felder P: La Santé autrement dit des methods alternatives éprouvées. En collaboration avec Johanna Crivelli-Roth. Yverdon-les-Bains, Editions Citrine, 1990.

50 Felder P: Erfahrungen mit der Naturheilkunde. Gesammelte Vorträge. Arlesheim, Yerbasana, 1997

51 Weber B: Bewährte Heilkräuter, die vor Deinem Hause wachsen. Nachdruck. Solothurn, Pauline Felder, kein Datum.

52 Stadelmann I: Die Hebammensprechstunde. Ermengerst, Stadelmann, 2005
53 Kelner M, Hall O, Coulter I: Chiropractors: Do they help? Toronto, Fitzhenry \& Whiteside, 1980.

54 Beebe J: Rapid Assessment Process: An Introduction. Walnut Creek, CA, AltaMira, 2001.

55 Trotter RT, Needle RH, Goosby E, et al: A methodological model for rapid assessment, response, and evaluation: the RARE Program in Public Health. Field Methods 2001;13:127159

56 Walach H, Falkenberg T, Fønnebø V, Lewith G, Jonas WB: Circular instead of hierarchical methodological principles for the evaluation of complex interventions. BMC Med Res Methodol 2006;6:29.

57 Boon H, Macpherson H, Fleishman S, et al: Evaluating complex healthcare systems: a critique of four approaches. Evid Based Complement Alternat Med 2007;4:279-285.

58 Eardley S, Bishop FL, Prescott P, et al: A systematic literature review of complementary and alternative medicine prevalence in EU. Forsch Komplementmed 2012;19(suppl 2): 18-28.

59 Uehleke B, Hopfenmueller W, Stange R, Saller R: Are the correct herbal claims by Hildegard von Bingen only lucky strikes? A new statistical approach. Forsch Komplementmed 2012;19:187-190.

60 Will H: Vergleich der Indikationen des «Kleinen Destillierbuches» des Chirurgen Hieronymus Brunschwig (Straßburg 1500) mit den nach derzeitigem wissenschaftlichem Erkenntnisstand belegten Indikationen. Dissertation zur Erlangung des naturwissenschaftlichen Doktorgrades der Julius-MaximiliansUniversität, Würzburg. Würzburg, 2009.

61 Freyer HPM: Europäische Heilkräuterkunde. Ein Erfahrungsschatz aus Jahrtausenden. Würzburg, Könighausen \& Neumann, 1998.

62 Stoll U, Müller B: Alte Rezepte modern betrachtet. Ein Versuch zur Beurteilung frühmittelalterlicher Pharmakotherapie aus heutiger Sicht. Geschichte der Pharmazie 1990;42: 33-40.

63 De Vos P: European materia medica in historical texts: longevity of a tradition and implications for future use. J Ethnopharmacol 2010; 132:28-47.

64 Smith M, Burton A, Falkenberg T: World Health Organization Traditional Medicine Strategy 2014-2023. New strategy for traditional and complementary medicine includes the development and use of herbal medicinal preparations. HerbalEGram 2014;11(5).

65 WHO Traditional Medicine Strategy. www. who.int/medicines/publications/traditionalpolicy/en/index.htm (aufgerufen am 24.03.2013).

66 Saller R: Evidenzbasiert - Ein Bedrohungsszenario für die Komplementärmedizin? Schweiz Zschr Gansheitsmedizin 2006;18:113. 